Article

\title{
Non-Fourier Heat Transfer with Phonons and Electrons in a Circular Thin Layer Surrounding a Hot Nanodevice
}

\author{
Vito Antonio Cimmelli *, Isabella Carlomagno and Antonio Sellitto \\ Department of Mathematics, Computer Science and Economics, University of Basilicata, Potenza \\ 85100, Italy; E-Mails: isabellacarlomagno85@gmail.com (I.C.); ant.sellitto@gmail.com (A.S.) \\ * Author to whom correspondence should be addressed; E-Mail: vito.cimmelli@ unibas.it; \\ Tel.: +39-0971-205885; Fax: +39-0971-205896.
}

Academic Editors: Michel Feidt, Daniel Tondeur, Jean-Noël Jaubert and Romain Privat

Received: 28 May 2015 / Accepted: 20 July 2015 / Published: 24 July 2015

\begin{abstract}
A nonlocal model for heat transfer with phonons and electrons is applied to infer the steady-state radial temperature profile in a circular layer surrounding an inner hot component. Such a profile, following by the numerical solution of the heat equation, predicts that the temperature behaves in an anomalous way, since for radial distances from the heat source smaller than the mean-free path of phonons and electrons, it increases for increasing distances. The compatibility of this temperature behavior with the second law of thermodynamics is investigated by calculating numerically the local entropy production as a function of the radial distance. It turns out that such a production is positive and strictly decreasing with the radial distance.
\end{abstract}

Keywords: temperature hump; nonlocal model for heat transfer; heat conduction with phonons and electrons

PACS Classifications: 44.10.+i; 66.70.-f; 05.70.Ln

\section{Introduction}

Nanotechnology has changed our vision, expectations and abilities to control all of the material world. It offers a huge amount of potential applications and solutions that in the recent past were only possible in the realm of scientific fiction. Its usefulness has been known for many years, especially 
in microelectronics and, more recently, in manufacturing of nano-electromechanical-systems (NEMS): systems with less space, material, energy consumption and usually higher performance can be actually realized. In particular, current research in nanoscaled electronic devices focuses the attention on carbon nanotubes, quantum wires and quantum dots, where the additional confinement of electrons is used to make compact high-performance components.

From the theoretical point of view, the use of nanotechnologies requires revising some well-known theories, as for example the classical Fourier law. Experimental evidence, in fact, clearly shows that it is completely inefficient to describe accurately heat transport at the nanometer length scale [1-7]. Several theories have been developed to describe heat transport in nanostructured materials [8-15].

In order to analyze thermoelectric effects, in [16] the authors developed a hydrodynamic model accounting for the possibility that both electrons and phonons may contribute to the heat transport. In particular, they assumed that the local heat flux $\mathbf{q}$ just arises from two contributions: the partial heat flux $\mathbf{q}_{p}$ due to the phonons and the partial heat flux $\mathbf{q}_{e}$ due to the electrons, in such a way that:

$$
\mathbf{q}=\mathbf{q}_{p}+\mathbf{q}_{e}
$$

The aforementioned model can be applied to describe situations in which both a voltage at the ends of a conductor is created by a temperature difference (Seebeck effect) and a heat flux is generated by an electric current (Peltier effect). However, for common materials, these direct conversions are not always efficient, since they only show limited thermoelectric properties, i.e., very small values of the Seebeck and Peltier material parameters. In such a case, for example, the Seebeck effect is only able to generate an electric current that is negligible, and in the absence of nonlinear effects, the evolution of the partial heat fluxes $\mathbf{q}_{p}$ and $\mathbf{q}_{e}$ in Equation (1) is ruled by the following equations:

$$
\begin{gathered}
\tau_{p} \dot{\mathbf{q}}_{p}+\mathbf{q}_{p}=-\lambda_{p} \nabla T+\ell_{p}^{2}\left(\nabla^{2} \mathbf{q}_{p}+2 \nabla \nabla \cdot \mathbf{q}_{p}\right) \\
\tau_{e} \dot{\mathbf{q}}_{e}+\mathbf{q}_{e}=-\lambda_{e} \nabla T+\ell_{e}^{2}\left(\nabla^{2} \mathbf{q}_{e}+2 \nabla \nabla \cdot \mathbf{q}_{e}\right)
\end{gathered}
$$

wherein $\left(\tau_{p} ; \ell_{p} ; \lambda_{p}\right)$ and $\left(\tau_{e} ; \ell_{e} ; \lambda_{e}\right)$ are the relaxation time, the mean-free path and the thermal conductivity of phonons and electrons, respectively, and $T$ is the absolute temperature. These equations account for nonlocal effects through the mean-free paths $\ell_{p}$ and $\ell_{e}$, and for memory effects through the relaxation times $\tau_{p}$ and $\tau_{e}$. They are still far from the standard form of the equations of linear heat-conduction theory with nonlocal and memory effects [17], since they contain information on the partial heat fluxes, relaxation times, thermal conductivities and mean-free paths.

The system above must be coupled to the local balance of energy, which, in the absence of heat sources and with negligible electric current, reads:

$$
c_{v} \dot{T}+\nabla \cdot \mathbf{q}=0
$$

with $c_{v}$ the specific heat of the conductor.

Although more general situations, allowing for different temperatures for phonons and electrons, could be considered $[18,19]$, in the present paper, we limit ourselves to the case in which the equations above hold. As we will see later, this assumption allows us to conserve a sufficient generality and reduces the calculations to a simpler level. Under the hypotheses above, here we study the radial heat transfer 
from a hot source surrounded by a circular layer and point out some non-standard characteristics of the temperature behavior.

The paper displays the following layout.

In Section 2, we evaluate the system of Equations (2) and (3) in steady states, in order to obtain the differential equation for the temperature profile. In the special case of a two-dimensional system with a radial symmetry, that equation is solved numerically, and the influence of nonlocal effects on the solution is pointed out.

In Section 3, we investigate the thermodynamic compatibility of the results obtained in Section 2 by calculating numerically the local entropy production as a function of the distance from the heat source. We show that such a quantity is positive everywhere in the system, according to the prediction of the second law of thermodynamics.

In Section 4, we draw the main conclusions and discuss possible developments of the present investigation.

\section{Radial Heat Transfer from a Hot Source}

In integrated circuits, the number of transistors that can be placed on a silicon chip has increased exponentially, since the developments in nanotechnology allow the transistors to shrink in size. With more transistors integrated on a single chip, running at a faster clock rate, designers have been able to improve the system performance effectively over the past few decades. Unfortunately, the design complexity, as well as the increase in the computational rate enhances the amount of produced heat per unit volume greatly, which has to be dissipated, thus being a limiting factor in current computer miniaturization. Being able to remove the surplus of produced heat may be of crucial interest in practical applications. Therefore, in the present section, we analyze the possible consequences of accounting for nonlocal effects in Equation (2). In particular, we assume that a small transistor (sketched as a hot cylindrical device) acts as a steady heat source and derive the temperature profile in the circular surrounding zone (the radius of which is $r_{l}$ ), wherein both the phonons and the electrons are the heat carriers (see Figure 1 for a qualitative sketch of the system we are considering). In order to emphasize the physical problem, we further assume that the inner hot device can only exchange heat with the surrounding circular layer. This is tantamount to assume that the system at hand (hot device and circular layer) is thermally isolated.

\subsection{The Physical Model}

In what follows, we represent the surrounding layer as a homogeneous and isotropic two-dimensional system and denote by $r$ the radial distance of its points from the center of the hot component (see Figure 1). Since we do not have information on the constitutive nature of the hot spot, in principle, we cannot exclude that heat propagates anisotropically inside it. Thus, there will be a thin annular region, starting at $r=r_{0}$, in which the transition from anisotropic to isotropic propagation may occur. For the sake of simplicity, we suppose that the dimension of this thin annulus is negligible and that the isotropic propagation starts at $r=r_{0}$. Thus, in steady states, the heat flux in the circular layer has only the radial component $q=q(r)$. 

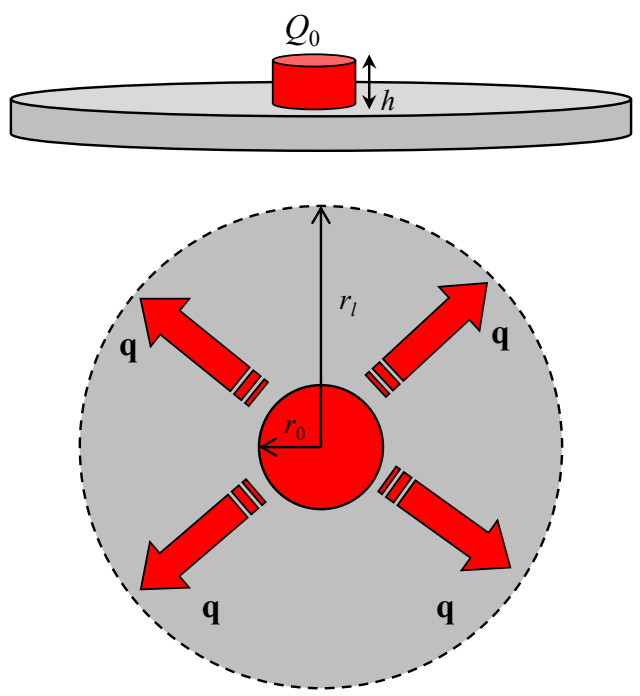

Figure 1. Cylindrical nanodevice connected to a circular surrounding layer. The internal device (i.e., the red hot spot in the figure) is characterized by a radius $r_{0}$ and a thickness $h$. It acts as a steady heat source, with $Q_{0}$ being the total heat produced per unit time. In the circular hot spot, we assumed that only the phonons are the heat carriers. The surrounding layer (grey in the figure) is characterized, instead, by an outer radius $r_{l}$. In it, both the phonons and the electrons contribute to the heat transfer. Outside that layer, instead, the electrons stop being the heat carriers again.

To obtain the radial dependence of $q$, one may observe that in the absence of transversal exchanges, the total heat flowing across each concentric circular area is constant. Therefore, by considering two different concentric circular areas at a radial distance from the source equal to $r$ and $r+d r$, the radial profile of the local heat flux (in a first-order approximation in $d r$ ) must be such that:

$$
2 \pi r q(r)=2 \pi[r+d r] q(r+d r) \approx 2 \pi[r+d r]\left[q(r)+\frac{d q}{d r} d r\right] \Rightarrow r \frac{d q}{d r}+q(r)=0
$$

The last relation in Equation (4) may be easily integrated and yields:

$$
q(r) \equiv q_{p}(r)+q_{e}(r)=\frac{\Gamma}{r}
$$

with $\Gamma=Q_{0} /(2 \pi h)$ as a constant value, being $Q_{0}$ the constant heat produced by the hot device per unit time.

In steady states, from Equations (1) and (3), we get:

$$
\nabla \cdot\left(\mathbf{q}_{p}+\mathbf{q}_{e}\right)=0
$$

On the other hand, since in our approach, $\mathbf{q}_{p}$ and $\mathbf{q}_{e}$ are independent state variables [4,16], the condition $\nabla \cdot \mathbf{q}_{p}=-\nabla \cdot \mathbf{q}_{e}$ cannot hold in general, but can only describe a particular situation. In the general case, instead, from Equation (6), it follows that both vectors $\mathbf{q}_{p}$ and $\mathbf{q}_{e}$ are divergence free. As a consequence, Equation (2) reduces to:

$$
\begin{aligned}
& \mathbf{q}_{p}=-\lambda_{p} \nabla T+\ell_{p}^{2} \nabla^{2} \mathbf{q}_{p} \\
& \mathbf{q}_{e}=-\lambda_{e} \nabla T+\ell_{e}^{2} \nabla^{2} \mathbf{q}_{e}
\end{aligned}
$$


which, by means of Equation (5), produces the following evolution equation for the phonon contribution to the local heat flux:

$$
\left(\frac{\lambda_{e}}{\lambda_{p}} \ell_{p}^{2}+\ell_{e}^{2}\right) \frac{d^{2} q_{p}}{d r^{2}}+\frac{1}{r}\left(\frac{\lambda_{e}}{\lambda_{p}} \ell_{p}^{2}+\ell_{e}^{2}\right) \frac{d q_{p}}{d r}-\left(\frac{\lambda_{e}}{\lambda_{p}}+1\right) q_{p}=\frac{\Gamma}{r}\left(\frac{\ell_{e}^{2}}{r^{2}}-1\right)
$$

This second-order differential equation can be solved by prescribing two Dirichlet-type boundary conditions. In fact, if we assume that both in the internal device $\left(r<r_{0}\right)$ and outside of the circular layer $\left(r>r_{l}\right)$ the phonons are the sole heat carriers, and since in steady states, $\Gamma$ takes a constant value, Equation (5) yields the following boundary conditions:

$$
\begin{aligned}
& q_{p}\left(r_{0}\right)=\frac{\Gamma}{r_{0}} \\
& q_{p}\left(r_{l}\right)=\frac{\Gamma}{r_{l}}
\end{aligned}
$$

Once the $q_{p}$ profile (and consequently, from Equation (5), the $q_{e}$ profile, too) in the surrounding circular layer has been obtained, then the solution of the following first-order differential equation:

$$
\frac{d T}{d r}=\left(\frac{\ell_{p}^{2}}{\lambda_{p}}\right) \frac{d^{2} q_{p}}{d r^{2}}+\frac{1}{r}\left(\frac{\ell_{p}^{2}}{\lambda_{p}}\right) \frac{d q_{p}}{d r}-\frac{q_{p}}{\lambda_{p}}
$$

allows us to obtain the radial temperature behavior in the neighborhood of the hot device. In this case, the boundary condition:

$$
T\left(r_{0}\right)=T_{0}
$$

$T_{0}$ being the temperature of the hot device, is sufficient to obtain the radial temperature profile.

\subsection{Results for a $\mathrm{Bi}_{2} \mathrm{Te}_{3}$ Surrounding Layer}

In order to investigate the influence of nonlocal effects on the radial heat transfer in a practical situation, here, we assume that the circular surrounding layer in Figure 1 is made of a $p$-doped sample of $\mathrm{Bi}_{2} \mathrm{Te}_{3}$ at the average temperature of $300 \mathrm{~K}$. The values of the different material functions involved

\begin{tabular}{|c|c|c|c|c|c|}
\hline$\lambda_{e}\left(\mathrm{~W} \mathrm{~m}^{-1} \mathrm{~K}^{-1}\right)$ & $\lambda_{p}\left(\mathbf{W} \mathbf{m}^{-1} \mathbf{K}^{-1}\right)$ & $\ell_{e}(\mathbf{m})$ & $\ell_{p}(\mathbf{m})$ & $r_{0}(\mathrm{~m})$ & $r_{l}(\mathbf{m})$ \\
\hline 2.4 & 1.6 & $9.1 \times 10^{-10}$ & $3.0 \times 10^{-9}$ & $10^{-9}$ & $10^{-8}$ \\
\hline
\end{tabular}
in our problem are summarized in Table 1 [20,21]. In that table, we also quote the radius of the internal device (i.e., $r_{0}$ ) and that of the circular layer (i.e., $\left.r_{l}\right)$.

Table 1. Values of the different quantities involved in the problem of the radial heat transfer described by the differential Equations (8) and (10).

In principle, the solutions of Equations (8) and (10) are strictly related to the particular experimental situations one is trying to analyze, as for example the amount of $Q_{0}$, as well as the value of $T_{0}$. In order to reduce these dependencies in the computation and to obtain results that are as general as possible, it is convenient to consider the following nondimensional version of Equation (8):

$$
\left(\alpha \mathrm{Kn}_{p}^{2}+\mathrm{Kn}_{e}^{2}\right) \frac{d^{2} q_{*}^{p}}{d r_{*}^{2}}+\frac{\left(\alpha \mathrm{Kn}_{p}^{2}+\mathrm{Kn}_{e}^{2}\right)}{r_{*}} \frac{d q_{*}^{p}}{d r_{*}}-(\alpha+1) q_{*}^{p}=\frac{\beta}{r_{*}}\left(\frac{\mathrm{Kn}_{e}^{2}}{r_{*}^{2}}-1\right)
$$


wherein the subscript $*$ indicates the (unitary) nondimensional quantities in such a way that $q_{p}=q_{0} q_{*}^{p}$, $r=r_{0} r_{*}$, and:

(1) $\alpha=\frac{\lambda_{e}}{\lambda_{p}}$

(2) $\mathrm{Kn}_{p}=\frac{\ell_{p}}{r_{0}}$

(3) $\mathrm{Kn}_{e}=\frac{\ell_{e}}{r_{0}}$

(4) $\beta=\frac{Q_{0}}{2 \pi h r_{0} q_{0}}$

Moreover, since, for the sake of computation, we assumed $r_{l}=10 r_{0}$, the boundary conditions in Equations (9) become:

$$
\begin{aligned}
& q_{*}^{p}(1)=\beta \\
& q_{*}^{p}(10)=\frac{\beta}{10}
\end{aligned}
$$

In Figure 2, we plot the theoretical profile of $q_{*}^{p}$ arising from the solution of the boundary value problem in Equations (12) and (13), when the surrounding layer is made of a $p$-doped sample of $\mathrm{Bi}_{2} \mathrm{Te}_{3}$ at the average temperature of $300 \mathrm{~K}$. In the bottom side of the same figure, we also plot the theoretical behavior of $q_{*}^{e}$, arising from Equation (5), namely,

$$
q_{*}^{e}=\frac{1}{r_{*}}-q_{*}^{p}
$$

We refer to Table 1 for the different parameters involved in the problem, whereas in Equation (12), we have set:

$$
\beta=1
$$

as it naturally arises from Equation (9a).

From the analysis of Figure 2, it follows that in any point of the circular layer $q_{*}^{e}$ is always one order of magnitude smaller than $q_{*}^{p}$. This means that for the particular problem we are analyzing, the phonons yield the main contribution to the local heat flux q. It seems also worth observing that $q_{*}^{p}$ attains its maximum in correspondence to the heat source (i.e., when $r_{*}=1$ ) and its minimum when $\left.r_{*} \in\right] 8 ; 9\left[\right.$, whereas $q_{*}^{e}$ has the minimum when $\left.r_{*} \in\right] 1 ; 2\left[\right.$ and the maximum when $r_{*} \approx 6$. Although these features seem to be related to the boundary conditions used, it will be seen in Section 3 that they are also connected to the entropy production in the circular layer. Thus, a deeper investigation of the role of boundary conditions seems to be necessary. 

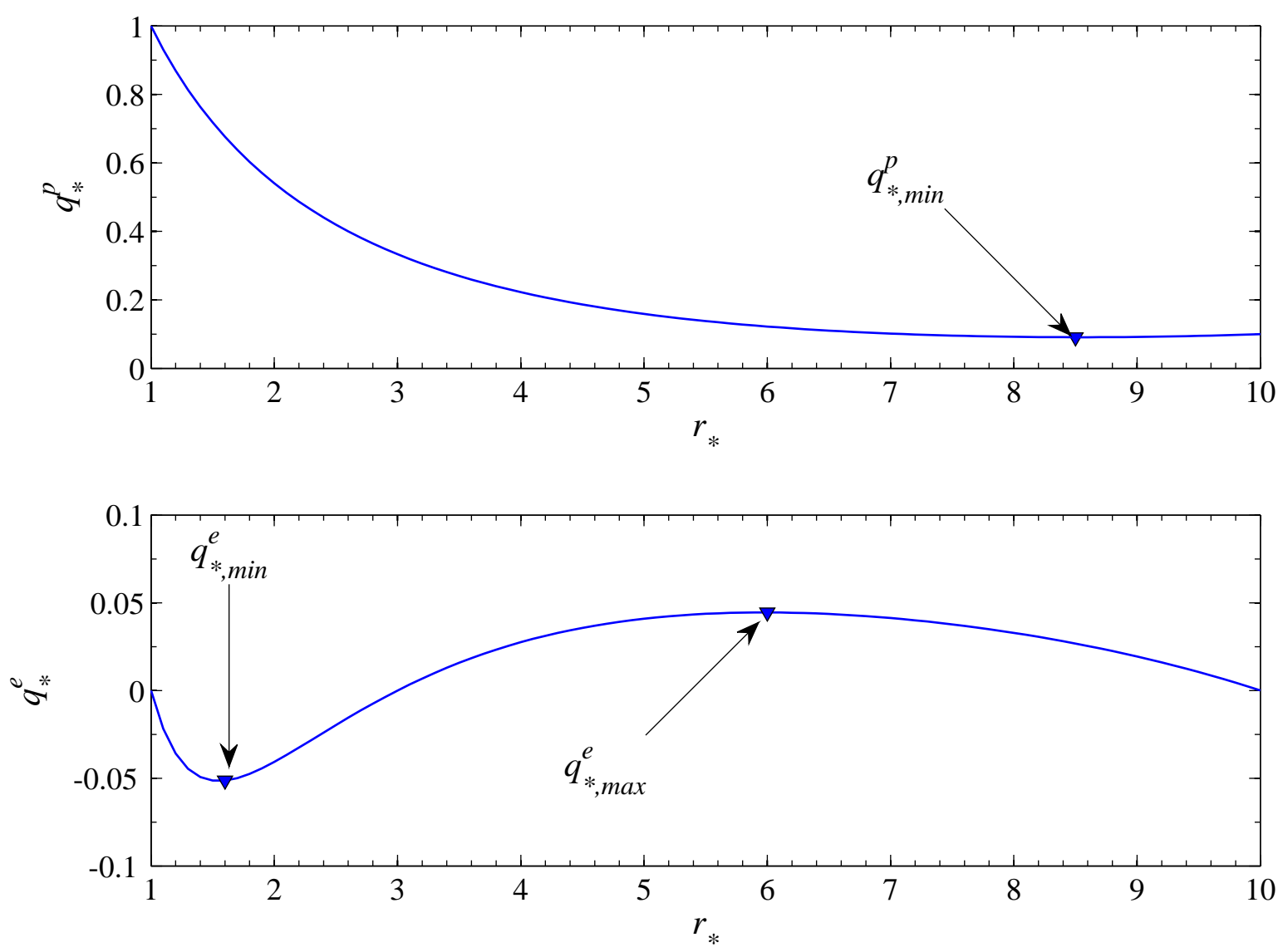

Figure 2. Behaviors of $q_{*}^{p}$ and $q_{*}^{e}$ versus the radial distance $r_{*}$ in the circular layer that surrounds the hot device. The profile of $q_{*}^{p}$ arises from the solution of the problem in Equation (12) with the boundary condition (13). The $q_{*}^{e}$ profile, instead, follows from Equation (5). Refer to Table 1 for the different parameters involved in the problem.

The results obtained for the heat-flux profiles can be used to pursue our goal, namely, to obtain the temperature profile in the neighborhood of the hot device. In particular, we can insert the previous result for $q_{*}^{p}$ in the following nondimensional version of Equation (10):

$$
\gamma \frac{d T_{*}}{d r_{*}}=\mathrm{Kn}_{p}^{2} \frac{d^{2} q_{*}^{p}}{d r_{*}^{2}}+\mathrm{Kn}_{p}^{2}\left(\frac{1}{r_{*}}\right) \frac{d q_{*}^{p}}{d r_{*}}-q_{*}^{p}
$$

wherein $T_{*}=T / T_{0}$ and $\gamma=\lambda_{p} T_{0} /\left(r_{0} q_{0}\right)$. Recalling that in the inner hot device, only the phonons may contribute to the heat transfer, it is easy to understand that $q_{0}$ should be proportional to $T_{0}, \lambda_{p}$ and $r_{0}$. These considerations allow us to claim that:

$$
\gamma=1
$$

in Equation (15). Then, its coupling to the nondimensional version of the boundary condition (11), i.e.,

$$
T_{*}(1)=1
$$

produces the result of Figure 3. 


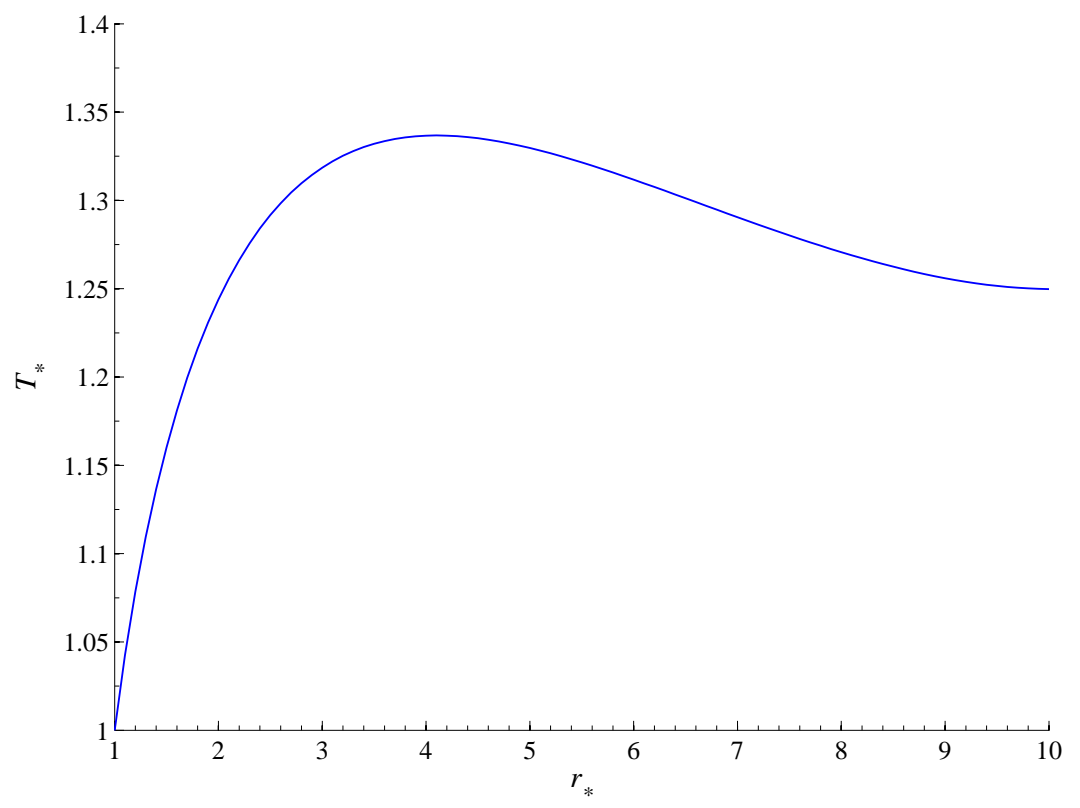

Figure 3. Behavior of $T_{*}$ versus the radial distance $r_{*}$ in the circular layer that surrounds the hot device. The theoretical profile for $T_{*}$ arises from the solution of the problem in Equation (15) with the boundary condition (17). Refer to Table 1 for the different parameters involved in the problem.

As it can be seen from Figure 3, in the neighborhood of the hot device, the temperature profile increases for increasing radial distances. The anomalous hump in the temperature profile that appears in Figure 3 is strictly related to the role played by nonlocal effects, as it is clearly shown by the presence of the coefficients $\mathrm{Kn}_{p}$ in the right-hand side of the heat-transport Equation (2). These effects should be taken into account in practical applications [22]. In fact, if the surrounding layer acts as an insulator among the different components of a same chip, which have to be kept at different temperatures, for example, then the results plotted in Figure 3 show that great attention has to be paid in shortening its size. In particular, Equation (15) points out that the temperature at a radial distance $r_{*}=10$ is about 1.25-times higher than that of the hot device at $r_{*}=1$, and this value might be unacceptable.

\section{Thermodynamic Compatibility}

The behavior shown in Figure 3 is anomalous, since the temperature profile in the layer is not everywhere decreasing with the radial distance, as it would be expected from the classical Fourier law [23]. Since in the present problem, the only heat source is the hot device at $r_{*} \equiv 1$, that behavior (arising from the model Equation (7)) points out that in the circular layer, there are regions wherein the heat is flowing from colder points to hotter ones.

In order to check the physical admissibility of the results plotted in Figure 3, in the present section, we analyze them in view of the second law of thermodynamics. Therefore, let us consider the local balance of the entropy

$$
\dot{s}=-\nabla \cdot \mathbf{J}^{(s)}+\sigma^{(s)}
$$


with $s$ as the entropy per unit volume,

$$
\mathbf{J}^{(s)}=\frac{\mathbf{q}}{T}+\left(\frac{\ell_{p}^{2}}{\lambda_{p} T^{2}}\right) \nabla \mathbf{q}_{p} \cdot \mathbf{q}_{p}+\left(\frac{\ell_{e}^{2}}{\lambda_{e} T^{2}}\right) \nabla \mathbf{q}_{e} \cdot \mathbf{q}_{e}
$$

as the entropy flux in the absence of the electric-current density [19] and $\sigma^{(s)}$ as the entropy production per unit volume.

The second law of thermodynamics states that $\sigma^{(s)}$ has to be always non-negative along arbitrary thermodynamic processes [24]. Since in steady states from Equation (18), it follows $\nabla \cdot \mathbf{J}^{(s)} \equiv \sigma^{(s)}$, then the results of Figure 3 are admissible on physical grounds if, and only if, the divergence of the entropy flux is non-negative everywhere in the layer. Indeed, by using the previous results for $q_{*}^{p}, q_{*}^{e}$ and $T_{*}$, the occurrence of that condition may be easily checked once the following nondimensional version of the constitutive relation in Equation (19) is introduced:

$$
J_{*}^{(s)}\left(r_{*}\right)=\frac{\beta}{r_{*} T_{*}}+\left(\frac{\mathrm{Kn}_{p}^{2}}{\gamma}\right) \frac{d q_{*}^{p}}{d r_{*}} q_{*}^{p}+\left(\frac{\mathrm{Kn}_{e}^{2}}{\gamma}\right) \frac{d q_{*}^{e}}{d r_{*}} q_{*}^{e}
$$

with $J^{(s)}=J_{*}^{(s)} q_{0} / T_{0}$.

Figure 4 plots the behavior of the nondimensional entropy production (i.e., the divergence of $J_{*}^{(s)}$ ) as a function of the radial distance from the hot device.

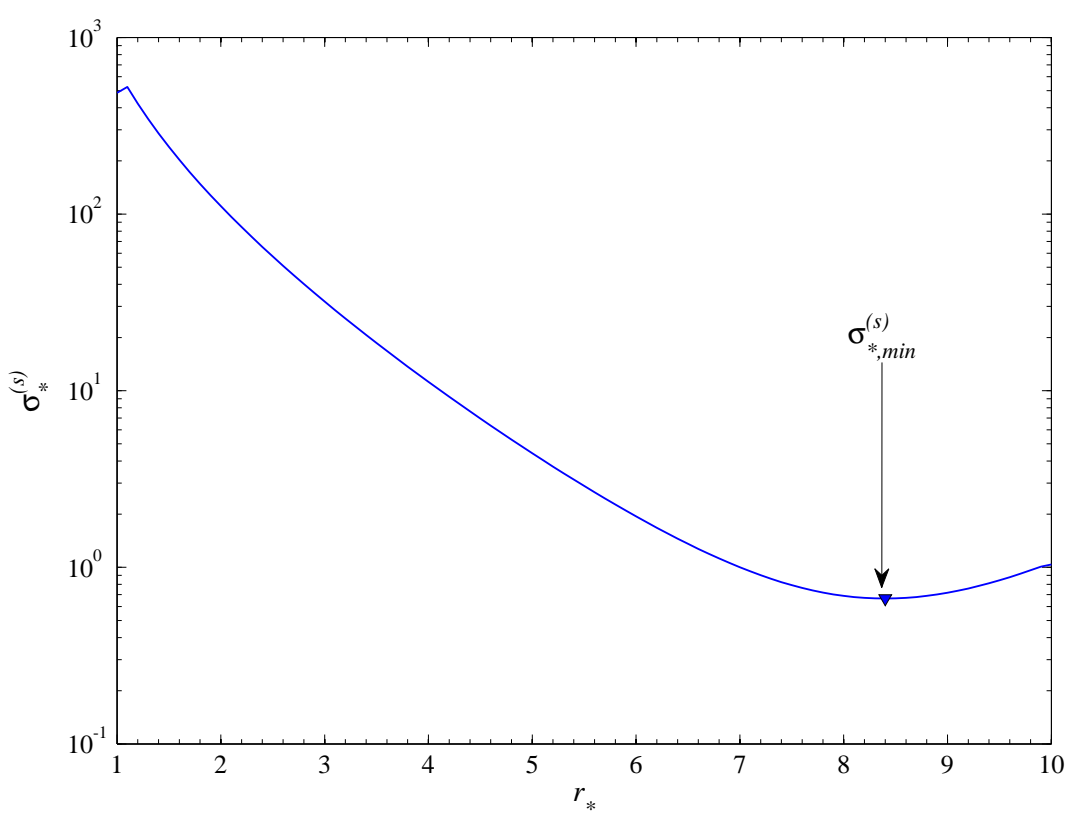

Figure 4. Behavior of $\sigma_{*}^{(s)}$ versus the radial distance $r_{*}$ in the circular layer that surrounds the hot device: theoretical results arising from the spatial derivatives of Equation (20). The $y$-axis in the figure is in a logarithmic scale.

As it can be seen from Figure 4, in the surrounding layer, $\sigma_{*}^{(s)}$ is everywhere positive. This proves that the hump in the temperature profile observed in Figure 3 is physically admissible, since it agrees with the second law of thermodynamics. 
In Figure 4, it is also possible to see that $\sigma_{*}^{(s)}$ qualitatively behaves as $q_{*}^{p}$ for increasing $r_{*}$, attaining its maximum and minimum at the same radial distances at which $q_{*}^{p}$ also reaches its maximum and minimum values, respectively. Moreover, $\sigma_{*}^{(s)}$ is increasing for $r_{*}>8.4$. We feel that this result is due to the principal role played by the phonon contribution to the local heat flux in the particular problem analyzed here, since it is logical to suppose that $\mathbf{J}^{(s)}$ is mainly influenced by $\mathbf{q}^{p}$. However, the behaviors observed in Figures 2 and 4 deserve deeper investigations, especially with respect to their possible connection to the boundary conditions.

\section{Conclusions}

In the present paper, we have applied the nonlocal model Equations (2) to describe heat transport in quasicrystalline materials [25], namely, in materials that should be properly located at the border line between metals and semiconductors [26]. For these materials, the heat transport is due to the combined flows of phonons and electrons [27], which may also have different temperatures [18,19]. Assuming that both the phonon temperature and the electron one coincide, in the case of steady-state radial heat transfer from an inner hot device surrounded by an external circular layer, we have analyzed the consequences on the temperature profile of the contribution of nonlocal terms entering the temperature equation. Due to the special role played by nonlocal effects at nanoscale, we observed that the temperature profile shows an anomalous hump in the neighborhood of the hot device. The presence of that hump (see Figure 3) suggests that there are some areas wherein the heat flows from colder points to the hotter ones. This result completely disregards that arising from the classical Fourier law [23], which predicts that heat can only flow from the hotter zones to the colder ones. However, to check whether the theoretical predictions arising from Equation (2) are physically admissible, or not, we have analyzed the temperature hump in view of the second law of thermodynamics. Figure 4 shows that the entropy production is everywhere positive and points out that the temperature hump is only an apparent violation of the basic principles of thermodynamics.

There are some further problems related to the present investigation, which deserve consideration and that will be analyzed in our future research.

As we noticed in the Section 1, it is also possible to consider more complex situations, with each of the heat carriers (phonons and electrons) endowed with its own temperature. Accounting for two different temperatures may be important, for instance, when a laser pulse hits the surface of a system. In such a case, initially, the electrons capture the main amount of the incoming energy with respect to the phonons. Subsequently, through electron-phonon collisions, they give a part of it to the phonons. As a further example, we mention those situations in which the electron mean-free path corresponding to electron-phonon collisions is long, so that one may have the so-called "hot electrons", namely, a population of electrons whose average kinetic energy (i.e., the kinetic temperature) is considerably higher than that of the phonons.

In the presence of two temperatures, the evolution of the partial heat fluxes $\mathbf{q}_{p}$ and $\mathbf{q}_{e}$ is governed by the following equations:

$$
\begin{gathered}
\tau_{p} \dot{\mathbf{q}}_{p}+\mathbf{q}_{p}=-\lambda_{p} \nabla T_{p}+\ell_{p}^{2}\left(\nabla^{2} \mathbf{q}_{p}+2 \nabla \nabla \cdot \mathbf{q}_{p}\right) \\
\tau_{e} \dot{\mathbf{q}}_{e}+\mathbf{q}_{e}=-\lambda_{e} \nabla T_{e}+\ell_{e}^{2}\left(\nabla^{2} \mathbf{q}_{e}+2 \nabla \nabla \cdot \mathbf{q}_{e}\right)
\end{gathered}
$$


wherein $T_{p}$ and $T_{e}$, respectively, mean the phonon and the electron contribution to the average temperature $T$ of the system [28]. Both $T_{p}$ and $T_{e}$ may be related to the internal energy per unit volume of phonons $u_{p}$ and to the internal energy per unit volume of electrons $u_{e}$, respectively, by means of the constitutive equations [28]:

$$
\begin{aligned}
& c_{v}^{p} T_{p}=u_{p} \\
& c_{v}^{e} T_{e}=u_{e}
\end{aligned}
$$

with $c_{v}^{p}$ being the specific heat at constant volume due to the phonons, and $c_{v}^{e}$ being the specific heat at constant volume due to the electrons, whereas the specific heat at constant volume of the whole system $c_{v}$ is given by $c_{v}=c_{v}^{p}+c_{v}^{e}$.

From the practical point of view, Equations (21) and (22) may lead to some perplexities, since one may naturally wonder whether $T_{p}$ and $T_{e}$ are measurable quantities, or not [29,30]. Here we notice that in [28] two possible strategies to measure those temperatures have been proposed.

Once Equations (22) have been assumed and both the specific heats are supposed to be constant, then the following partial energy balances can be postulated:

$$
\begin{aligned}
& c_{v}^{p} \dot{T}_{p}+\nabla \cdot \mathbf{q}_{p}=0 \\
& c_{v}^{e} \dot{T}_{e}+\nabla \cdot \mathbf{q}_{e}=0
\end{aligned}
$$

For temperature-dependent specific heats, Equations (23) become nonlinear. However, such a nonlinearity, which is due to the coefficients of the time derivatives of the partial temperatures, does not influence the temperature profile in steady states. Recalling that whenever both electrons and phonons contribute to the heat flow, the internal energy per unit volume $u$ of the whole system is given by $u=u_{p}+u_{e}$, then the use of Equation (22) allows us to relate $T_{p}$ and $T_{e}$ to the average temperature $T$ of the system as:

$$
T=\frac{c_{v}^{p} T_{p}+c_{v}^{e} T_{e}}{c_{v}}
$$

once the thermodynamic relation $u=c_{v} T$ is used. Note further that the summation of Equation (23) produces the local energy balance (3).

The combination of Equations (21) with Equations (23) allows us, in principle, to derive the temperatures behaviors analytically, which may be interesting in practical applications. However, the solution of that problem is much more complex with respect to the case considered here. In [19], the authors analyzed the consequences of accounting for Equations (21) and (23) in steady-state radial heat transfer from a point source when the partial contributions to the local heat flux are such that:

$$
\begin{aligned}
\mathbf{q}_{p} & =\left(\frac{\lambda_{p}}{\lambda_{p}+\lambda_{e}}\right) \mathbf{q} \\
\mathbf{q}_{e} & =\left(\frac{\lambda_{e}}{\lambda_{p}+\lambda_{e}}\right) \mathbf{q}
\end{aligned}
$$

In this special case, both $T_{p}$ and $T_{e}$ display anomalous behaviors, which, however, are in accordance with the second law of thermodynamics [19]. 
Relaxing the constitutive assumptions (25), it would be interesting to obtain a numerical solution for both the temperature profiles $T_{p}(r)$ and $T_{e}(r)$, in order to infer the different role played by phonons and electrons in quasicrystalline materials.

Another interesting problem is related to the efficiency of the thermoelectric coupling to two temperatures. In the presence of thermoelectric effects, the system of Equations (21)-(23) needs to be completed by suitable balance equations for the electric charge and for the electric current (see $[18,19]$ for more details). Such a problem, when the nonlocal effects may be neglected, has been analyzed in [28] with a uniform chemical potential due to the electric charge density and in [31] with a nonuniform chemical potential due to the electric charge density. From those references, it follows that for practical applications, in order to enhance the efficiency of the thermoelectric energy conversion, it would be useful to have:

(1) the charge distribution as homogeneous as possible;

(2) the ratio $T_{e} / T$ as high as possible.

In our future research, we aim to extend the previous investigation to the case in which also nonlocal effects are taken into account, in order to check how these effects may influence the efficiency of thermoelectric devices.

\section{Acknowledgments}

Isabella Carlomagno acknowledges the financial support of the University of Basilicata and of the University of Salento.

Vito Antonio Cimmelli acknowledges the support of the Italian Gruppo Nazionale per la Fisica Matematica (GNFM-INdAM) and of the University of Basilicata.

The contribution of Antonio Sellitto to the present work was supported by the National Group of Mathematical Physics (GNFM-INdAM).

The results contained in the present paper have been presented at the "13th Joint European Thermodynamics Conference" (JETC 2015), held in Nancy, France, from 20 May to 22 May 2015.

The authors gratefully acknowledge the Organizing Committee of JETC 2015 for the opportunity for several interesting discussions on new trends in non-equilibrium thermodynamics.

\section{Author Contributions}

In the preparation of this article, all three authors were fully involved in: substantial conception and design of the paper; drafting the article and revising it critically for important intellectual content; final approval of the version to be published. All authors have read and approved the final manuscript.

\section{Conflicts of Interest}

The authors declare no conflict of interest. 


\section{References}

1. Chen, G. Nanoscale Energy Transport and Conversion-A Parallel Treatment of Electrons, Molecules, Phonons, and Photons; Oxford University Press: Oxford, UK, 2005.

2. Zhang, Z.M. Nano/Microscale Heat Transfer; McGraw-Hill: New York, NY, USA, 2007.

3. Ferry, D.K.; Goodnick, S.M. Transport in Nanostructures, 2nd ed.; Cambridge University Press: Cambridge, UK, 2009.

4. Jou, D.; Casas-Vázquez, J.; Lebon, G. Extended Irreversible Thermodynamics, 4th ed.; Springer: Berlin, Germany, 2010.

5. Volz, S., Ed. Thermal Nanosystems and Nanomaterials; Springer: Berlin/Heidelberg, Germany, 2010.

6. Cahill, D.G.; Braun, P.V.; Chen, G.; Clarke, D.R.; Fan, S.; Goodson, K.E.; Keblinski, P.; King, W.P.; Mahan, G.D.; Majumdar, A.; et al. Nanoscale thermal transport. II. 2003-2012. Appl. Phys. Rev. 2014, $1,011305$.

7. Lee, S.; Broido, D.; Esfarjani, K.; Chen, G. Hydrodynamic phonon transport in suspended graphene. Nat. Commun. 2015, 6, 6290.

8. Chen, G. Ballistic-diffusive heat-conduction equations. Phys. Rev. Lett. 2001, 86, 2297-2300 .

9. Alvarez, F.X.; Jou, D.; Sellitto, A. Phonon hydrodynamics and phonon-boundary scattering in nanosystems. J. Appl. Phys. 2009, 105, 014317.

10. Wang, M.; Yang, N.; Guo, Z.Y. Non-Fourier heat conductions in nanomaterials. J. Appl. Phys. 2011, 110, 1064310.

11. Tzou, D.Y. Nonlocal behavior in phonon transport. Int. J. Heat Mass Transf. 2011, 54, 475-481.

12. Lebon, G.; Jou, D.; Dauby, P.C. Beyond the Fourier heat conduction law and the thermal non-slip condition. Phys. Lett. A 2012, 376, 2842-2846.

13. De Tomas, C.; Cantarero, A.; Lopeandia, A.F.; Alvarez, F.X. Thermal conductivity of group-IV Semiconductors from a Kinetic-Collective Model. Proc. R. Soc. A 2014, 470, 20140371.

14. Kazan, M.; Masri, P. The contribution of surfaces and interfaces to the crystal thermal conductivity. Surf. Sci. Rep. 2014, 69, 1-37.

15. Kovács, R.; Ván, P. Generalized heat conduction in heat pulse experiments. Int. J. Heat Mass Transf. 2015, 83, 613-620.

16. Jou, D.; Cimmelli, V.A.; Sellitto, A. Nonlocal heat transport with phonons and electrons: Application to metallic nanowires. Int. J. Heat Mass Transf. 2012, 55, 2338-2344 .

17. Lebon, G.; Jou, D.; Casas-Vázquez, J. Understanding Non-Equilibrium Thermodynamics; Springer: Berlin, Germany, 2008.

18. Jou, D.; Sellitto, A.; Cimmelli, V.A. Phonon temperature and electron temperature in thermoelectric coupling. J. Non-Equilib. Thermodyn. 2013, 38, 335-361.

19. Jou, D.; Sellitto, A.; Cimmelli, V.A. Multi-temperature mixture of phonons and electrons and nonlocal thermoelectric transport in thin layers. Int. J. Heat Mass Transf. 2014, 71, 459-468.

20. Satterthwaite, C.B.; Ure, R.W., Jr. Electrical and thermal properties of $\mathrm{Bi}_{2} \mathrm{Te}_{3}$. Phys. Rev. 1957, $108,1164-1170$. 
21. Qui, B.; Sun, L.; Ruan, X. Lattice thermal conductivity reduction in $\mathrm{Bi}_{2} \mathrm{Te}_{3}$ quantum wires with smooth and rough surfaces: A molecular dynamics study. Phys. Rev. B 2011, 83, 035312.

22. Sellitto, A.; Alvarez, F.X. Non-Fourier heat removal from hot nanosystems through graphene layer. Nanoscale Syst. Math. Model. Theory Appl. 2012, 1, 38-47 .

23. Sellitto, A.; Jou, D.; Bafaluy, J. Nonlocal effects in radial heat transport in silicon thin layers and graphene sheets. Proc. R. Soc. A 2012, 468, 1217-1229.

24. Cimmelli, V.A.; Jou, D.; Ruggeri, T. Ván, P. Entropy Principle and Recent Results in Non-Equilibrium Theories. Entropy 2014, 16, 1756-1807 .

25. Maciá, E. Theoretical prospective of quasicrystals as thermoelectric materials. Phys. Rev. B 2001, 64, 094206.

26. Tamura, R.; Waseda, A.; Kimura, K.; Ino, H. Semiconductorlike transport in highly ordered Al-Cu-Ru quasicrystals. Phys. Rev. B 1994, 50, 9640(R).

27. Lin, Z.; Zhigilei, L.V.; Celli, V. Electron-phonon coupling and electron heat capacity of metals under conditions of strong electron-phonon nonequilibrium. Phys. Rev. B 2008, 77, 075133.

28. Sellitto, A.; Cimmelli, V.A.; Jou, D. Influence of electron and phonon temperature on the efficiency of thermoelectric conversion. Int. J. Heat Mass Transf. 2015, 80, 344-352.

29. Berciaud, S.; Han, M.Y.; Mak, K.F.; Brus, L.E.; Kim, P.; Heinz, T.F. Electron and Optical Phonon Temperatures in Electrically Biased Graphene. Phys. Rev. Lett. 2010, 104, 227401.

30. Schreier, M.; Kamra, A.; Weiler, M.; Xiao, J.; Bauer, G.E.W.; Gross, R.; Goennenwein, S.T.B Magnon, phonon, and electron temperature profiles and the spin Seebeck effect in magnetic insulator/normal metal hybrid structures. Phys. Rev. B 2013, 88, 094410 .

31. Rogolino, P.; Sellitto, A.; Cimmelli, V.A. Influence of the electron and phonon temperature and of theelectric-charge density on the optimal efficiency of thermoelectric nanowires. Mech. Res. Commun. 2015, doi:10.1016/j.mechrescom.2015.03.002.

(c) 2015 by the authors; licensee MDPI, Basel, Switzerland. This article is an open access article distributed under the terms and conditions of the Creative Commons Attribution license (http://creativecommons.org/licenses/by/4.0/). 